\title{
Possibility of Estimating the Risk of Falling at the Seat-Off Face While Rising Up from a Chair
}

\author{
Hideo Hirai ${ }^{1 *}$, So Miyahara ${ }^{1}$ and Seiji Togashi ${ }^{2}$ \\ ${ }^{1}$ Faculty of Health Sciences, Hiroshima Cosmopolitan University, Hiroshima, Japan \\ ${ }^{2}$ Professor and Dean of Graduate School at Hiroshima Cosmopolitan University, Japan
}

*Corresponding author: Hideo Hirai, Associate Professor at Department of Rehabilitation, Hiroshima Cosmopolitan University, Japan, Email: hirai@hcu.ac.jp

\section{Research Article}

Volume 4 Issue 1

Received Date: January 13, 2021

Published Date: February 04, 2021

DOI: $10.23880 /$ aabsc- 16000157

\section{Abstract}

It is known that falls in the daily life of the elderly occur frequently during walking and rising up and greatly affect their quality of life. In the previous study we obtained the COG-heel horizontal distance and the COG horizontal velocity when the subjects performed rising up under the "fastest" or "slowest" velocity task. These seat-off parameters which were an indicator of the stability in rising from a chair were defined based on the relationship between them. In this experiment with healthy adults as subjects the seat-off parameters were found to become a different distribution because of the rising up strategies of each subject. Moreover, based on the relationship between the seat-off parameters and motor function, they could become an index expressing current fall risk and predicting future fall risk.

Keywords: Rising up motion; Seat-off; COG-heel horizontal distance; COG horizontal velocity; Fall risk

\section{Introduction}

Rising up motion is a systemic and antigravity motor function that humans acquire as a pre-stage of walking ability in the process of motor development. In order to perform the rising up motion, the posture control mechanism accompanying the development and maturation of the central nerve system must be normally constructed and motor commands from the central nerve system need to be accurately reflected through the peripheral nerve system. With these backgrounds, various rising up motion patterns depending on the environment and circumstances are performed, and motor functions that have very important meaning which cannot be avoided in daily life of human beings are demonstrated. However if the ability to control one's body is impaired due to aging or disease, the risk of falls is significantly increased. So far research on rising up motion has been conducted under various conditions for elderly and disabled patients. Most of these studies were related to the responsiveness of rising up motion to changes in the external environment [16]. On the other hand, in the case of falls in actual situations, elderly people tend to fall when trying to rise up in a hurry or when trying to rise up slowly. There are caused by changes in the internal environment due to psychological background and physiological needs. These things suggest that falls in the daily life of elderly people are not only caused by changes in the external environment, but can also be attributed to unusual rising up motion speeds that occur in response to the internal environment and posture control strategies based on it [7-11]. In our previous study, a seat-off parameter was created using a motion capture system and a floor reaction force meter to evaluate the balance ability of an elderly group with unstable rising up and an elderly group with stable rising up during rising up. As a result, the elderly group that showed instability in rising up was divided into two groups: an anterior imbalance group and a posterior imbalance group [12]. In this study we did the same as in previous experiments for a young group and examined their seat-off distribution. In addition the experiment measured functional reach, cadence during fastest walking, and knee extension strength, and investigated the relationship between them and seat-off parameters. 


\section{Methods}

\section{Subjects}

The subjects consisted of eight healthy males and four healthy females aged 20. All subjects had no disease that interfered with rising up motion. Prior to the experiment all participants received a description of the experiment and were provided written informed consent. The study was approved by the ethics review committee of Hiroshima Cosmopolitan University.

Rising up motion: A chair without an armrest was used for the rising up motion test, and the seat height was the length of the lower leg of the subject. The starting position is a sitting posture with the trunk held as vertical as possible and the hip, knee, and ankle joints defined as $90^{\circ}$, and the ending position is a stationary rising posture with the head and trunk held vertically. In addition both hands were placed in front of the chest and both feet were stationary. Immediately before starting the rising up motion, either one of the two tasks "fastest" or "slowest" is randomly taught, and the rising up motion from the chair was performed five times each and a motion capture system (Move-tr/3D, library) to acquire and analyze image data was used. In the rising up motion, the fastest rising up motion possible for each individual was defined as the fastest value, and the slowest rising up motion possible for each individual was defined as the slowest value. Each subject was instructed not to move their feet during rising up movement. The sampling rate for image analysis was $50 \mathrm{fps}$, and the marker positions are parietal, binaural foramen, bilateral ridges, lateral epicondyles of both humerus, bilateral pedicles, right third fingertip, bilateral trochanter, central part of both knee gaps, both limbs The fifth metatarsal head. In addition to these marker points, the head point, chest point, and waist point were defined as follows in order to calculate the body center of gravity.

- Head point: midpoint of the left and right ear canal markers

- Chest point: midpoint of left and right shoulder markers

- Lumbar point: midpoint of the greater trochanter marker on the left and right

- The timing of seat-off was determined by the video data captured by the analysis software. COG-heel horizontal distance at the moment of seat-off was defined as the horizontal distance between a part of the supporting basal plane malleolus lateralis and the body's center of gravity. COG horizontal velocity was defined as the average speed of the preceding frame and one following frame, which were calculated.

Motor function measurement: In addition to the subject's height, weight, sitting height and thigh length, as motor functions, functional reach indicating dynamic balance ability, walking cadence indicating the number of steps per unit time (fastest walking), and maximum isometric knee extension muscle strength (left and right) were used as variables to investigate the relationship with rising up motion [13]. The functional reach was measured three times according to the method of Duncan et al and the maximum value was adopted [14]. The walking cadence was measured three times according to the method of Kinugasa et al and the maximum number of steps was adopted [15]. Knee extension muscle strength is measured using the self-powered exercise training device (ISO force GT-330, OG Giken, Japan), measuring the maximum isometric knee extension muscle strength three times for each of the left and right legs, and the average value of each maximum value was defined as the maximum isometric knee extension muscle strength.

\section{Analysis Method}

The COG-heel horizontal distance and COG horizontal velocity during the execution of the "fastest" and "slowest" risingup tasks were calculated, and (1) to (4) shown in Figure 1 were the analysis items. (1): width of COG-heel horizontal distance (2): width of COG horizontal velocity (3): slowestfastest distance $\sqrt{(1)^{2}+(2)^{2}}$. (4): slowest-fastest inclination (2)/(1)). In addition, we plotted the seat-off parameter (described later) of all subjects on the coordinates with the horizontal axis with a width of COG-heel distance and the vertical axis with a width of COG horizontal velocity. We used this to infer the possibility of a fall risk. We also evaluated the relationship between the four analyzed items and motor function by Spearman's correlation coefficient.

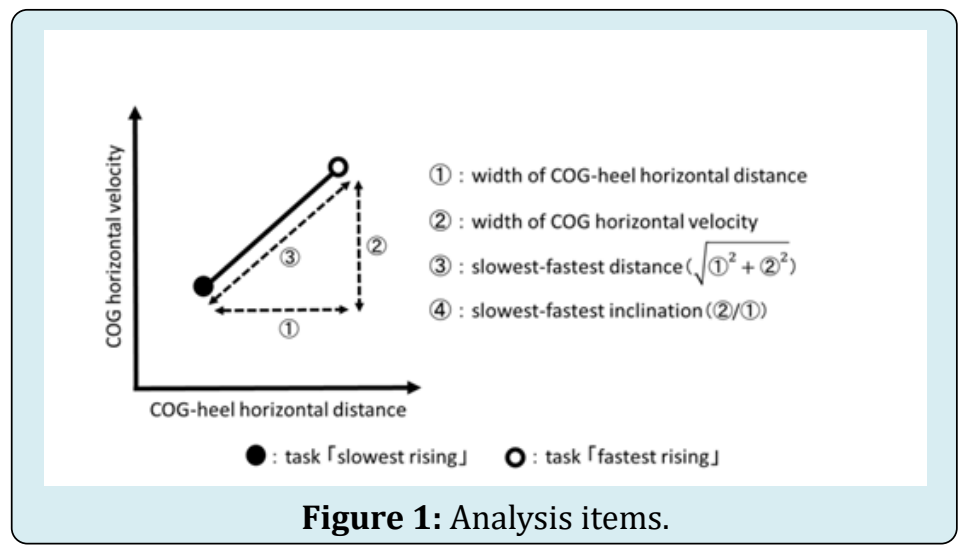

Hideo Hirai, et al. Possibility of Estimating the Risk of Falling at the Seat-Off Face While Rising Up from a Chair. Ann Adv Biomed Sci 2021, 4(1): 000157. 


\section{Results}

\section{Seat-off parameter}

Figure 2 shows the COG-heel horizontal distance, COG horizontal velocity, slowest-fastest distance, and of the slowest-fastest inclination of seat-off when 12 subjects were rising. In all cases, there is a positive correlation between the
COG-heel horizontal distance and the COG horizontal velocity, but the length and slope of each are different for each subject. Therefore, width of COG-heel horizontal distance and width of COG horizontal velocity of all subjects were normalized and they were plotted on the coordinates with (1) COG heel horizontal distance width as the horizontal axis and (2) COG horizontal velocity width as the vertical axis (Figure. 3). In this study, (1) and (2) were defined as seat-off parameters.

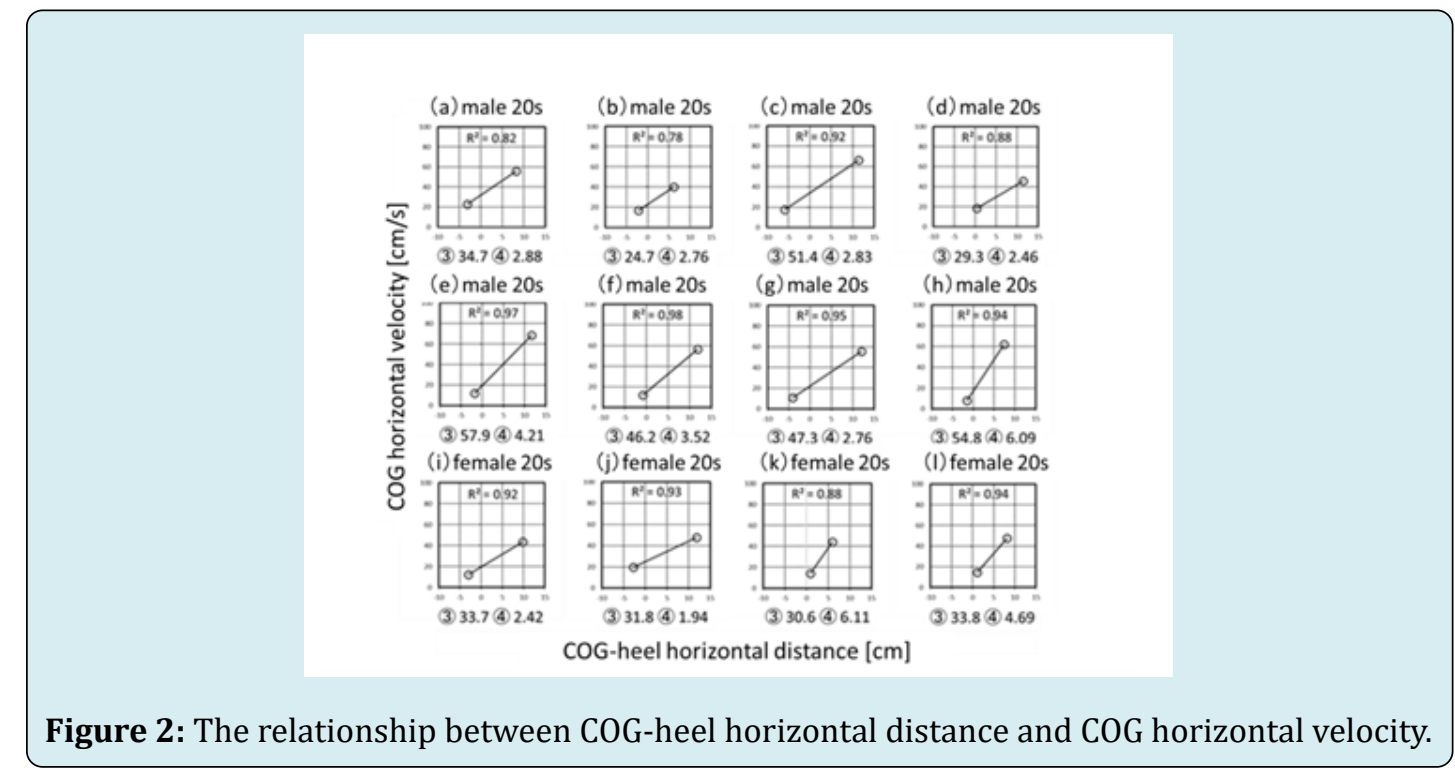

Figure 2: The relationship between COG-heel horizontal distance and COG horizontal velocity.

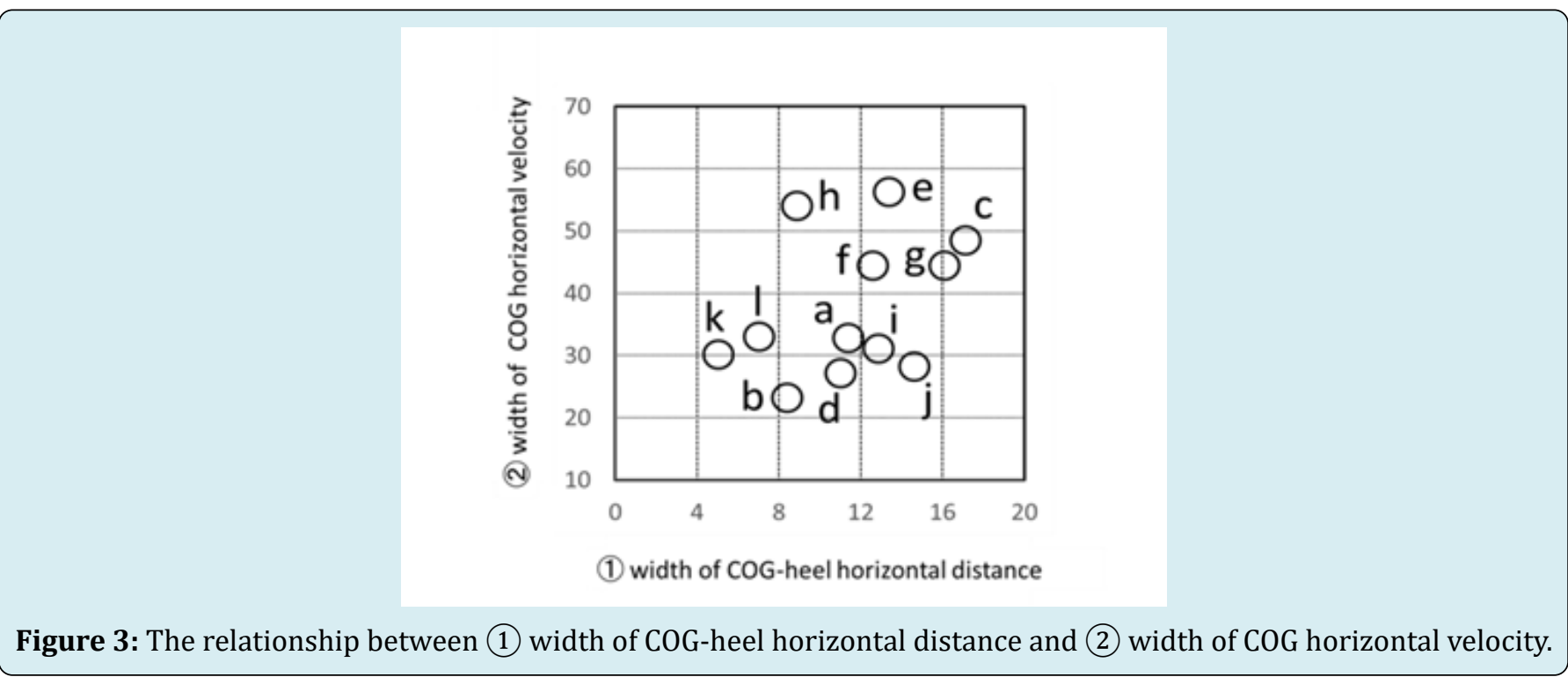

\section{Motor function}

Table 1 shows the measurement results of the subject's motor function, and Table 2 shows the correlation between the seat-off parameter and motor function. (1) the width of
COG-heel horizontal distance and functional reach was 0.84 , and the fastest walking cadence was 0.89 . The correlation coefficient between (2) the width of COG horizontal velocity and maximum isometric knee extension muscle strength was 0.85 . 


\begin{tabular}{|c|c|c|c|c|}
\hline Subjects & Age, gender & Functional reach [cm] & $\begin{array}{c}\text { Cadence at fastest } \\
\text { walking [step/min] }\end{array}$ & $\begin{array}{c}\text { Maximum isometric knee } \\
\text { extension muscle strength[n] }\end{array}$ \\
\hline a & $20 \mathrm{~s}, \mathrm{male}$ & $37.7 \pm 0.3$ & $142.0 \pm 0.2$ & $734 \pm 110$ \\
\hline b & $20 \mathrm{~s}, \mathrm{male}$ & $38.1 \pm 0.8$ & $147.6 \pm 0.5$ & $356 \pm 67$ \\
\hline c & $20 \mathrm{~s}, \mathrm{male}$ & $39.3 \pm 1.3$ & $168.6 \pm 0.1$ & $718 \pm 103$ \\
\hline d & $20 \mathrm{~s}, \mathrm{male}$ & $30.4 \pm 0.7$ & $134.3 \pm 0.3$ & $454 \pm 110$ \\
\hline e & $20 \mathrm{~s}$, male & $39.4 \pm 0.5$ & $169.6 \pm 0.5$ & $1008 \pm 104$ \\
\hline f & $20 \mathrm{~s}$, male & $41.8 \pm 1.9$ & $157.3 \pm 0.4$ & $876 \pm 147$ \\
\hline g & $20 \mathrm{~s}$, male & $40.8 \pm 1.8$ & $143.9 \pm 0.3$ & $1047 \pm 93$ \\
\hline h & $20 \mathrm{~s}$, male & $40.3 \pm 1.6$ & $164.5 \pm 0.3$ & $766 \pm 112$ \\
\hline i & $20 \mathrm{~s}, \mathrm{female}$ & $37.5 \pm 0.7$ & $152.3 \pm 0.3$ & $775 \pm 109$ \\
\hline j & $20 \mathrm{~s}$, female & $36.9 \pm 0.3$ & $167.3 \pm 0.6$ & $757 \pm 87$ \\
\hline k & $20 \mathrm{~s}$, female & $33.1 \pm 0.5$ & $135.6 \pm 0.2$ & $458 \pm 112$ \\
\hline I & $20 \mathrm{~s}$, female & $33.7 \pm 0.4$ & $141.3 \pm 1.0$ & $480 \pm 80$ \\
\hline
\end{tabular}

Table 1: Motor function of all subjects.

\begin{tabular}{|c|c|c|c|}
\hline & Functional reach & Cadence at fastest walking & $\begin{array}{c}\text { Maximum isometric knee } \\
\text { extension muscle strength }\end{array}$ \\
\hline $\begin{array}{c}\text { (1) width of COG-heel horizontal } \\
\text { distance }\end{array}$ & 0.84 & 0.89 & 0.30 \\
\hline (2) width of COG horizontal velocity & 0.54 & 0.32 & 0.85 \\
\hline
\end{tabular}

$p$ value $<0.01$

Table 2: The relationship between seat-off parameter and motor function.

\section{Discussion}

Normally, the consciousness of rising up from the sitting position is reflected in the lifting motion as the center of gravity movement speed based on the posture control strategy, and the rising up motion is performed. Therefore, the relationship between the COG-heel horizontal distance and the COG horizontal velocity during seat-off reflects the characteristics of the rising style and is considered to be one of the important factors that determine whether to fall or not fall. From the results of the rising up motion experiment of the elderly by Ikeda et al., It is pointed out that the loss of balance during this task is reflected in falls in daily life [16]. Schenkman, et al. also said that the forward momentum of the upper body during rising up motion was converted upward [17]. Furthermore, in order to not fall while rising, it is necessary to be able to perform rising up motions suitable for the environment and to have smoothness of the rising up motion itself. In other words, the stability of rising up motion is considered to be brought by adaptability and smoothness.

In this study, "adaptiveness" is considered to correspond to (3) slowest-fastest distance, which is the distance of the line segment formed by two seat-off parameters corresponding to both "slowest" and "fastest" tasks. If this distance is shortened, the range of the seat-off parameter that enables rising is narrowed, so the adaptability to the rising up motion according to the situation becomes low. Conversely, if this distance is long, it means that the adaptability to the rising up motion becomes high. "Smoothness" is considered to correspond to the slope of the line segment between two seat-off parameters due to the strength of the dependence of the attitude control strategy that is exhibited in response to the "slowest" and "fastest" tasks. In other words, "smoothness" was considered to correspond to (4) the slowest-fastest inclination. Therefore, when taking a posture control strategy that depends on the dynamic balance ability, the width of COG-heel horizontal distance becomes larger, the slowest-fastest inclination becomes moderate, and conversely, the posture control strategy depends on the lower limb muscle strength. In this case, it is assumed that the width of COG horizontal velocity is large and the slowestfastest inclination tends to be steep. Figure 4 plots the 12 subjects shown in Figure 3 within coordinates with (1) the width of the COG heel horizontal distance on the horizontal axis and (2) the width of the COG horizontal velocity on the vertical axis. This is a schematic diagram assuming the risk of the subject falling due to changes in the width of the horizontal distance and the width of the horizontal velocity of each seat-off parameter. 


\section{Distribution of seat-off parameters}

As shown in Figure 3, the distribution of the seat-off parameters of all 12 subjects had a high positive cor-relation within a certain range of variation. From this, it can be judged that the "slowest" and "fastest" rising up motion tasks of this time were rising by a similar attitude control strategy. In other words, the responsiveness to the difference in the rising up speed required in this experiment is obtained by con-trolling the position of the center of gravity at the seatoff face and changing the width of the support base. Judging from this result, all subjects were healthy and had no fall history, the distribution range of the seat-off parameter in these healthy groups showed stability in the seat-off face. If there is a sign that deviates from the distribution range, it may suggest that the posture control strategy depends on either dynamic balance ability or lower limb strength, it tries to cope with the task by changing the position of the center of gravity as a posture control strategy, but the COG horizontal velocity that is the driving force ahead of the center of gravity cannot be obtained. Therefore, it is assumed that (1) the width of COG-heel horizontal distance becomes wider and its inclination becomes moderate. On the other hand, if it depends too much on the muscle strength of the lower limbs, the posture control strategy tries to cope with the task with COG horizontal velocity, which is the propulsive force forward of the center of gravity, but the change the width of the support base is reduced. Therefore, (2) the width of COG horizontal velocity will increase and the slope will tend to be steep. In Figure 4, this hypothesis is illustrated, divided into types A to D according to the characteristics of the seat-off parameter, and the specific assumed fall was verified. First, it can be interpreted that Type A, which has a tendency to have a large (1) width of COG-heel horizontal distance and (2) the width of COG horizontal velocity, have both high adaptability and smoothness (inclination) in rising up motion. On the other hand, Type B, which tends to be small in (1) the width of COG-heel horizontal distance and (2) the width of COG horizontal velocity, is less adaptable to rising up motions, but has smoothness within a limited range. Type A and Type B are bipolar, but both have the characteristic that (1) and (2) correspond to each other. Therefore, subjects who fall within this range are considered to have a low risk of falling in their daily lives. So, it can be inferred that the distribution of the seat-off parameter of the 12 subjects in Figure 3 is located somewhere between the type A and the type B. Therefore, the dotted elliptical region in Figure 4 is a stable region of rising up motion within its own adaptation range, and as it deviates from this distribution region, the stability becomes lower, meaning that the risk of falling is increased. On the other hand, in the case of type C in Figure 4, (1) the width of COG-heel horizontal distance is smaller and (2) the width of COG horizontal velocity is larger than type A and type B, so (3) the slowest-fastest distance is longer and (4) the slowest-fastest inclination is steep. In other words, because of (1) the width of COG-heel horizontal distance is narrow and (2) the width of COG horizontal velocity increases, the rising up motion is not smooth. In particular, the tendency to fall forward at a rising up motion is expected to increase. This analysis may support the report by Buatois, et al. That the predicted probabilities of recurrent falls were correlated with the speed of rising up as well as the fall history [18]. Also, type D is a type with (1) the width of COG-heel horizontal distance is wide and (2) the width of COG horizontal velocity is narrow, so (3) the slowest-fastest distance is long and (4) the slowest-fastest inclination becomes gentle. Elderly people with balance disorders take longer to stand up than subjects without balance disorders [19]. This is thought to be the case when the signs of type D had progressed. Since (1) the width of COG-heel horizontal distance in-creases and (2) the width of COG horizontal velocity decreases, it is expected that the rising up motion will not be smooth and in particular the tendency to fall backwards will increase.

\section{The relationship between seat-off parameters and motor function}

It is considered that the risk of falls can be reduced by complementing the motor function caused by the distribution range of the seat-off parameter, and by grasping the motor ability and instructing the acquisition of the rising up style that matches the individual motor ability. Optimal cut-off points for standard measurements of lower limb function in the elderly are said to help identify elderly who are not disabled but are at high risk of developing the disorder [20]. Type C, which is considered to be at risk of falling, was predicted to have a particularly high tendency to fall forward. In this case, by increasing the width of the horizontal distance, it is possible to correct the low tendency of (3) the slowestfastest distance and the steep tendency of (4) the slowestfastest inclination. As a result, the excessive forward force can be converted into an upward force, which may suppress the tendency of falling forward and improve the stability during the rising up motion. In the results of this experiment, the width of the horizontal distance showed a strong positive correlation with the functional reach and the cadence during the fastest walking. In the case of the type $\mathrm{C}$, which is thought to be the cause of instability due to the narrow horizontal distance at the seat-off face, by enhancing these motor skills, the narrowness of the horizontal distance at the seat-off face may be corrected and the risk of falls may be improved. In addition, it is necessary to devise a way to move the COG upward during daily rising up movements and instruction to correct excessive speed of rising up motion. In the type D, the tendency to fall backward was expected. In this case, if the width of the horizontal velocity is extended, it is possible to correct the low tendency of (3) the slowest-fastest distance and the gentle tendency of (4) the slowest-fastest inclination. 
As a result, the excessive upward force can be converted into a forward force, which may suppress the tendency of falling backward and improve the stability during the rising up motion. In the results of this experiment, the width of COG horizontal velocity showed a strong correlation with the maximum isometric knee extension muscle strength. In the case of type $\mathrm{D}$, the narrowness of the horizontal velocity at the seat-off face was considered to be the cause of instability. By increasing the maximum isometric knee extension muscle strength, the narrowness of the horizontal velocity of the seat-0ff face may be corrected and the fall risk of rising up may be improved. In addition, it is necessary to devise a way to move the COG forward during daily rising up movements and instruction to increase the rising up motion speed is required. All the subjects who participated in this experiment have physical abilities that do not interfere with their daily lives, so there is almost no possibility of falling. Therefore, their seat-off parameters were distributed near the center of Figure 4, reflecting the stability of their rising up motion. When this distribution area deviated in the type $\mathrm{C}$ or type $\mathrm{D}$ direction due to various factors, it was found that the risk of falling of rising up motion was increased, as described in the previous experimental results of the elderly group [12].

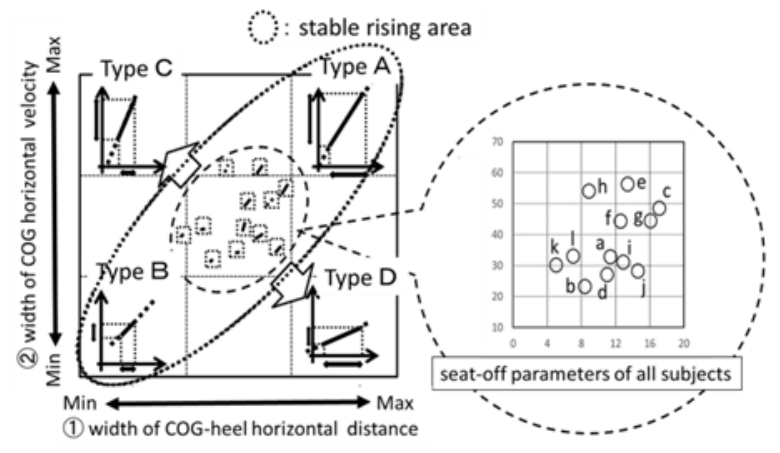

Figure 4: Distribution of standing motion strategy by seat-off parameter.

\section{Conclusion}

The rising up motion and motor ability of 12 healthy adults aged 20 to 64 years were measured, and the seat-off parameters and motor function data were calculated. As a result, the following became clear.

1) Width of COG-heel horizontal distance and width of COG horizontal velocity were defined as seat-off parameters.

2) Width of COG-heel horizontal distance showed a strong positive correlation with functional reach and cadence during fastest walking.

3) COG horizontal velocity was strongly correlated with maximum isometric knee extension strength.

4) Based on the seat-off parameter, the distribution range of the rising up motion at the seat-off face was obtained and the risk of falling of rising up motion was considered.

\section{References}

1. Numazawa $S$, Sato $Y$, Saito A, Inoue K, Katagiri T, et al. (2003) Verification of classified situations for falls of elderly people in nursing homes. Yamagata Journal of Health Science 6: 64-68.
2. Kozuki
K, Konishi
R, Higuchi
Y, Takeuchi

M, Nishimura Y, et al. (2010) Prognostic validity of executive function and injurious fall history in evaluating injurious fall risk among frail elderly people. Journal of rehabilitation and health sciences 8: 23-28.

3. Yamada T, Demura S, Kitabayashi T (2004) Influence of seat height on floor reaction force and strength of lower limbs during sit-to-stand movement. Japan Society of Physiological Anthropology 9(2): 47-52.

4. Taniuchi K (2007) Influences of different chair seating postures, that effects rising movements and rising postures. Japanese journal of occupational medicine and traumatology 55(2): 85-94.

5. Mori A, Eguchi A, Watanabe A (2003) Influence of the differences in chair height of rising up and sitting down movement on electromyographic activities in lower extremity muscles. Kawasaki Medical Welfare Society 13: 169-171.

6. Hoshi F, Yamanaka M, Takahashi M, Maki M (1995) The Sit-to-Stand motion pattern and posturography in elderly people. Hokkaido University Collection of Scholarly and Academic Papers 8: 81-88 
7. Maruta K (2004) The Influence of Seat Angle on Forward Trunk Inclination During Sit-to-Stand. Physical Therapy Japan 31(1): 21-28.

8. Serita T, Maruyama H, Kato M, Suganuma K, Sakakibara $\mathrm{R}$, et al. (2011) Investigation into the Relationships between the Trunk and Lower Leg Inclination and Speed in Rising up from a Chair and Sitting Down. Rigakuryoho Kagaku 26(4): 483-488.

9. Yokoyama H, Aoki U, Kasahara M, Hiraki K (2004) Relationship between isometric knee extension strength and ability to rise from a chair in elderly patients. Rigakuryoho Kagaku 31(2): 106-112.

10. Tatewaki T, Kaneko S (2005) A study of control mechanism for of rising-up achieving a motion task. Rigakuryoho Kagaku 20(4): 303-307.

11. Schenkman ML, Berger RA, Riley PO (1990) Whole-body movements during rising to rising from sitting: Physical Therapy 70(10): 638-648.

12. Hirai H, Oka $H$ (2016) Evaluation of the balance capabilities of elderly people rising in the longitudinal direction. Advanced Biomedical Engineering 5: 13-18.

13. Arai T, Shiba Y, Watanabe S, Shibata H (2011) The relationship between the stride time variability motor ability and Fal1 in community-dwelling elderly people. Rigakuryoho Kagak 38(3): 165-172.

14. Duncan PW, Winter DK, chandler J, Studenski S (1990) Functional reach: a new clinical measure of balance.
45(6): 192-197.

15. Kinugasa T, Nagasaki H, Ito H, Hashizume K, Furuna T, et al. (1994) Effect of aging on motor ability in men aged 18 to 83 years. Phys Fitness Sports Med 43: 343-351.

16. Elizabeth R Ikeda, Margaret L Schenkman, Patrick O Riley, W Andrew Hodge (1991) Influence of Age on Dynamics of Rising from a Chair. Physical Therapy 71(6): 473-481.

17. Margaret Schenkman, Richard A Berger, Patrick O Riley, Robert W Mann, W Andrew Hodge (1990) Whole-Body Movements During Rising to Standing from Sitting. Physical Therapy 70(10): 638-648.

18. Severine Buatois, Christine Perret-Guillaume, Rene Gueguen, Patrick Miget, Guy Vançon, et al. (2010) A Simple Clinical Scale to Stratify Risk of Recurrent Falls in Community-Dwelling Adults Aged 65 Years and Older. Physical Therapy 90(4): 550-560.

19. Susan L Whitney, Diane M Wrisley, Gregory F Marchetti, Michael A Gee, Mark S Redfern, et al. (2005) Clinical Measurement of Sit-to-Stand Performance in People with Balance Disorders. Validity of Data for the Five-TimesSit-to-Stand Test: Physical Therapy 85(10): 1034-1045,

20. Hyuma Makizako, Hiroyuki Shimada, Takehiko Doi, Kota Tsutsumimoto, Sho Nakakubo, et al. (2017) Predictive Cutoff Values of the Five-Times Sit-to-Stand Test and the Timed "Up \& Go" Test for Disability Incidence in Older People Dwelling in the Community. Physical Therapy. 97: 417-424. 